\title{
Prioridades de investigación para la arquitectura ecuatoriana desde el enfoque socioeconómico, tecnológico y educativo. Una experiencia Delphi
}

\section{Research priorities for Ecuadorian architecture from the socioeconomic, technological and educational approach. A Delphi experience}

Narváez-Ricaurte AM

Gómez-García AR

Universidad Internacional SEK, Ecuador

Autor por Correspondencia: alex.narvaez@uisek.edu.ec

Fecha de recepción: 18 de Abril de 2017 - Fecha de aceptación: 10 de Julio de 2017

Resumen: La investigación debe ser liderada por las instituciones de educación superior con el objeto de vincularse de manera significativa con los sectores estratégicos y productivos del país. El objetivo es identificar y priorizar los temas de investigación sobre la arquitectura ecuatoriana desde un enfoque socioeconómico, tecnológico y educativo. Material - Método: Consenso grupal de 15 docentes universitarios mediante la técnica Delphi, desarrollada en 2 fases consecutivas: (i) identificación de brechas y (ii) establecimiento de prioridades. Los resultados fueron ordenados en función de la mediana y desviación estándar. Se evidencia la necesidad de investigar aspectos tecnológicos en los procesos constructivos, sus afectaciones en las dinámicas socio-económicas y el impacto en el ambiente y sus ciclos naturales, como herencia del paso del tiempo en los territorios y la consolidación de ciudades. La universidad se convierte en el medio transmisor de la investigación para la generación del conocimiento, permitiendo a los profesionales dar respuestas oportunas y pertinentes a las necesidades de las ciudades, sus ciudadanos y los procesos económicos, ambientales, sociales y culturales.

Palabras clave: arquitectura; investigación; universidad; Delphi; ecuador

Abstract: Research must be led by institutions of higher education in order to be linked in a meaningful way with the strategic and productive sectors of the country. The objective is to identify and prioritize research topics on Ecuadorian architecture from a socioeconomic, technological and educational perspective. Material - Method: Consensus of 15 university teachers using the Delphi technique, developed in two consecutive phases: (i) identification of gaps and (ii) establishment of priorities. The results were ordered according to the median and standard deviation. It is evident the need to investigate technological aspects in the construction processes, their effects on the socio-economic dynamics and the impact on the environment and its natural cycles, as an inheritance of the passage of time in the territories and the consolidation of cities. The university becomes the transmitter of research for the generation of knowledge, allowing professionals to provide timely and relevant answers to the needs of cities, their citizens and economic, environmental, social and cultural processes.

Key words: architecture; research; university; Delphi; Ecuador 


\section{Introducción}

La investigación en Ecuador sigue siendo una de las grandes debilidades en todos sus ámbitos con respecto a otros países de Latinoamérica1. No obstante, la Ley Orgánica de Educación Superior de 2011 anima a las universidades a investigar para responder a las necesidades de la sociedad, tanto en la educación superior de grado y posgrado2-3.

Es por ello, que la investigación debe ser liderada por las instituciones de educación superior con el objeto de vincularse de manera significativa con los sectores estratégicos y productivos del país, existiendo una relación directa entre investigación, sociedad, avance tecnológico y desarrollo económico para que beneficie en el bienestar social4, aspecto estrechamente relacionado con el Plan Nacional para el Buen Vivir para el período de 2013 a 2017, que promueve la interacción recíproca entre la educación, el sector productivo y la investigación científica - tecnológica para la transformación de la matriz productiva y la satisfacción de las necesidades del país5.

En la actualidad existen diferentes metodologías para el establecimiento y priorización en investigación6, si bien no predomina un método especifico a nivel mundial, el más empleado ha sido la técnica Delphi, caracterizado por ser un proceso de comunicación grupal que permite definir y consensuar líneas de investigación mediante el empleo de la estadística para establecer un orden de importancia a las líneas plantadas7.

El objetivo del presente estudio fue identificar y priorizar los temas de investigación sobre la arquitectura ecuatoriana mediante la técnica Delphi, adoptando un enfoque socioeconómico, tecnológico y educativo.

\section{Material - método}

\section{Diseño}

Consenso de docentes de la Facultad Arquitectura e Ingenierías de la Universidad Internacional SEK en Ecuador mediante el empleo de la técnica Delphi8-9 desarrollada en 2 fases; (i) identificación de brechas y (ii) establecimiento de prioridades de investigación en arquitectura.

\section{Participantes}

Participaron 15 docentes a tiempo completo de la UISEK con experiencia en diversas áreas afines a la arquitectura y urbanismo; construcciones, dibujo, diseño arquitectónico y urbano, estructuras, topografía, rehabilitación urbana, historia y normativa de la arquitectura.

\section{Primera Fase}

En una primera fase, realizada el 4 de enero de 2017, dentro del I Taller sobre Investigación Científica en Arquitectura permitió introducir en el proceso de consenso posterior a los participantes. 
Una vez expuestos los objetivos de la reunión, el moderador planteó la pregunta (brecha) de cuáles deberían ser las líneas de investigación de arquitectura en el contexto ecuatoriano. De forma individual, cada participante fue anotando aquellas respuestas desde su experiencia personal y profesional durante un plazo de 30 minutos. Finalizado éste, se procedió a la lectura de las respuestas por docente, el moderador anotaba en la pizarra del aula para la visualización de todos.

Mediante Focus Group, se debatieron y clarificaron todas las respuestas. Finalmente, por consenso se identificaron un total de 10 brechas de interés en investigación para arquitectura, quedando descartadas 2 brechas al no estar relacionadas con esta área de conocimiento, Tabla 1.

Tabla 1. Listado de brechas de interés en investigación para arquitectura, Focus Group.

\begin{tabular}{ll}
\hline $\begin{array}{l}\text { Brecha } \\
\mathbf{N}^{\mathbf{o}}\end{array}$ & Denominación \\
\hline $1^{\text {o }}$ & Crítica de la arquitectura local \\
$2^{\text {o }}$ & Historia como instrumento en el proceso de arquitectura \\
$3^{\text {o }}$ & Dibujo como instrumento en el proceso de diseño \\
$4^{\text {o }}$ & Arquitectura como respuesta a las necesidades sociales-locales \\
$5^{\text {o }}$ & Intervención sobre el patrimonio arquitectónico \\
$6^{\text {o }}$ & Papel de la arquitectura en las catástrofes naturales \\
$7^{\text {o }}$ & Espacio público y arquitectura \\
$8^{\text {o }}$ & Desarrollo arquitectónico y urbano en procesos naturales \\
$9^{\text {o }}$ & Bases epistemológicas de la arquitectura en la educación superior \\
$10^{\text {o }}$ & Mejoramiento de procesos constructivos \\
\hline
\end{tabular}

\section{Segunda Fase}

Con objeto de priorizadas las brechas de investigación identificadas, la segunda fase, realizada el 11 de enero de 2017, consistió en administrar a los participantes los formularios que contenían el listado de brechas. Para cada una de las ellas, se solicitó a los participantes que asignaran una puntuación mediante una escala tipo Likert con valores que oscilaron de 0 a 5 $(0=$ sin interés; $1=$ podría tener algún interés; $2=$ poco importante; $3=$ moderadamente importante; 4=bastante importante; 5=muy importante) sobre la importancia, necesidad e impacto de investigar en lo referente al componente social, económico y tecnológico, igualmente, el impacto que supondría cada una de las brechas en la docencia (malla curricular de la carrera de arquitectura).

Puntadas cada una de las brechas y en función a los 4 componentes, se procedió a validar la fiabilidad de los resultados obtenidos mediante el Alfa de Cronbach, alcanzando una puntuación $\alpha=0,838$ para los 40 elementos analizados (>.8=bueno) 10 .

\section{Análisis}

Para el análisis de los datos se empeló el software Statistical Package for the Social Sciences (versión 23). Se presentan las frecuencias absolutas (n) y relativas (\%n), media ( $\square$ ), mínimo - máximo y desviación estándar (DE). 
Para establecer el orden de prioridad se consideró la mediana (calculada a partir de los promedios totales), siendo un valor estadístico que permite una buena aproximación al grado de prioridad consensuado por el grupo de docentes.

Finalmente, se presenta la descripción narrativa de las respuestas obtenidas en función de su mayor mediana de puntuación considerando la desviación estándar.

\section{Resultados}

En la Tabla 2 se presentan la distribución porcentual de las puntuaciones para cada brecha de investigación por componentes; docencia, social, económico y tecnológico.

\section{Docencia}

Las brechas sobre Crítica de la arquitectura local, Historia como instrumento en el proceso de arquitectura y Espacio público y arquitectura obtuvieron los porcentajes más altos $(\mathrm{n}=15 ; 100 \%)$, consideradas por el grupo de participantes como bastante ó muy importante, seguido de las Bases epistemológicas de la arquitectura en la educación superior ( $\mathrm{n}=14 ; 93,3 \%)$.

\section{Social}

En cuanto al componente social, destaca en primer lugar el Espacio público y arquitectura $(\mathrm{n}=15 ; 100 \%)$, seguido de la Arquitectura como respuesta a las necesidades sociales-locales y el Papel de la arquitectura en las catástrofes naturales ( $\mathrm{n}=14 ; 93,3 \%)$ y, Desarrollo arquitectónico y urbano en procesos naturales $(n=13 ; 86,7 \%)$ como bastante o muy importante.

\section{Económico}

Desde un enfoque económico, el Papel de la arquitectura en las catástrofes naturales $(\mathrm{n}=15 ; 100 \%)$ y Mejoramiento de procesos constructivos $(\mathrm{n}=14 ; 93,3 \%)$ son consideradas como brechas de investigación bastante ó muy importantes.

\section{Tecnológico}

Finalmente, en cuanto al impacto que supondría en el avance y desarrollo tecnológico como bastante ó muy importante, el Papel de la arquitectura en las catástrofes naturales $(\mathrm{n}=15$; $100 \%)$, Mejoramiento de procesos constructivos ( $\mathrm{n}=14 ; 93,3 \%)$ e Intervención sobre el patrimonio arquitectónico $(\mathrm{n}=13 ; 86,2 \%)$ fueron las brechas con puntuaciones más altas.

Respecto a las medias más altas y desviación estándar por brecha y componente destacan; la Crítica de la arquitectura local e Historia como instrumento en el proceso de arquitectura con una media de 4,87 $\pm 0,35$ en la docencia y el Papel de la arquitectura en las catástrofes naturales en el ámbito social $(4,67 \pm 0,62)$, económico $(4,73 \pm 0,46)$ y tecnológico $(4,80 \pm 0,41)$. 
Tabla 2. Distribución de las puntuaciones pro brecha de investigación.

\begin{tabular}{|c|c|c|c|c|c|c|c|c|c|c|c|c|c|c|c|c|c|}
\hline \multirow{2}{*}{$\begin{array}{l}\text { Puntuación } \\
\text { Brecha } \mathrm{N}^{0}\end{array}$} & \multicolumn{2}{|c|}{ Docencia } & \multicolumn{2}{|c|}{ Social } & \multicolumn{2}{|c|}{ Económico } & \multicolumn{2}{|c|}{ Tecnológico } & \multirow{2}{*}{$\begin{array}{l}\text { Puntuación } \\
\text { Brecha } \mathrm{N}^{0}\end{array}$} & \multicolumn{2}{|c|}{ Docencia } & \multicolumn{2}{|c|}{ Social } & \multicolumn{2}{|c|}{ Económico } & \multicolumn{2}{|c|}{ Tecnologgico } \\
\hline & $n$ & $\% n$ & $n$ & $\% n$ & $n$ & $\% n$ & $n$ & $\% n$ & & $n$ & $\% n$ & $n$ & $\% n$ & $n$ & $\%$ n & $n$ & $\%$ n \\
\hline $1^{0}$ & & & & & & & & & $6^{0}$ & & & & & & & & \\
\hline 0.1 & - & - & 2 & $13,30 \%$ & 3 & $20,00 \%$ & 5 & $33,30 \%$ & 0.1 & 1 & $6,70 \%$ & - & - & - & - & - & - \\
\hline 02-mar & - & - & 5 & $33,30 \%$ & 10 & $66,70 \%$ & 8 & $53,30 \%$ & 02-mar & 3 & $20,00 \%$ & 1 & $6,70 \%$ & - & - & - & - \\
\hline 04-may & 15 & $100 \%$ & 8 & $53,30 \%$ & 2 & $13,30 \%$ & 2 & $13,30 \%$ & 04-may & 11 & $73,30 \%$ & 14 & $93,30 \%$ & 15 & $100 \%$ & 15 & $100 \%$ \\
\hline $2^{0}$ & & & & & & & & & $7^{0}$ & & & & & & & & \\
\hline 0.1 & - & - & 2 & $13,30 \%$ & 3 & $20,00 \%$ & 4 & $26,70 \%$ & $0-1$ & - & - & - & - & - & - & 2 & $13,30 \%$ \\
\hline 02-mar & - & - & 4 & $26,70 \%$ & 8 & $53,30 \%$ & 7 & $46,70 \%$ & 02-mar & - & - & - & - & 6 & $40,00 \%$ & 9 & $60,00 \%$ \\
\hline 04-may & 15 & $100 \%$ & 9 & $60,00 \%$ & 4 & $26,70 \%$ & 4 & $26,70 \%$ & 04-may & 15 & $100 \%$ & 15 & $100,00 \%$ & 9 & $60,00 \%$ & 4 & $26,70 \%$ \\
\hline $3^{0}$ & & & & & & & & & $8^{0}$ & & & & & & & & \\
\hline 0.1 & - & - & 5 & $33,30 \%$ & 7 & $46,70 \%$ & 6 & $40,00 \%$ & 0.1 & - & - & - & - & - & - & - & - \\
\hline 02-mar & 3 & $20,00 \%$ & 9 & $60,00 \%$ & 7 & $46,70 \%$ & 6 & $40,00 \%$ & 02-mar & 3 & $20,00 \%$ & 2 & $13,30 \%$ & 5 & $33,30 \%$ & 4 & $26,70 \%$ \\
\hline 04-may & 12 & $80,00 \%$ & 1 & $6,70 \%$ & 1 & $6,70 \%$ & 3 & $20,00 \%$ & 04-may & 12 & $80,00 \%$ & 13 & $86,70 \%$ & 10 & $66,70 \%$ & 11 & $73,30 \%$ \\
\hline $4^{0}$ & & & & & & & & & $9^{0}$ & & & & & & & & \\
\hline 0.1 & 1 & $6,70 \%$ & - & - & - & - & 3 & $20,00 \%$ & 0.1 & 1 & $6,70 \%$ & 3 & $20,00 \%$ & 4 & $26,70 \%$ & 3 & $20,00 \%$ \\
\hline 02-mar & 3 & $20,00 \%$ & 1 & $6,70 \%$ & 3 & $20,00 \%$ & 6 & $40,00 \%$ & 02-mar & - & - & 7 & $46,70 \%$ & 7 & $46,70 \%$ & 7 & $46,70 \%$ \\
\hline 04-may & 11 & $73,30 \%$ & 14 & $93,30 \%$ & 12 & $80,00 \%$ & 6 & $40,00 \%$ & 04-may & 14 & $93,30 \%$ & 5 & $33,30 \%$ & 3 & $20,00 \%$ & 5 & $33,30 \%$ \\
\hline $5^{0}$ & & & & & & & & & $10^{\circ}$ & & & & & & & & \\
\hline 0.1 & - & - & - & - & - & - & - & - & 0.1 & - & - & - & - & - & - & - & - \\
\hline 02-mar & 4 & $26,70 \%$ & 3 & $20,00 \%$ & 4 & $26,70 \%$ & 2 & $13,30 \%$ & 02-mar & 2 & $13,30 \%$ & 4 & $26,70 \%$ & - & - & 1 & $6,70 \%$ \\
\hline 04-may & 11 & $73,30 \%$ & 12 & $80,00 \%$ & 11 & $73,30 \%$ & 13 & $86,70 \%$ & 04-may & 13 & $86,70 \%$ & 11 & $73,30 \%$ & 14 & $93,30 \%$ & 14 & $93,30 \%$ \\
\hline
\end{tabular}

$0=\sin$ interés; $1=$ podría tener algún interés; $2=$ poco importante; $3=$ moderadamente importante $; 4=$ bastante importante; $5=$ muy importante 
En cuanto al orden de prioridad consensuado por el grupo de docentes a través de las medianas globales, las principales brechas de interés en investigación son; el Papel de la arquitectura en las catástrofes naturales (Brecha $\mathrm{N}^{\circ} 6$ ) con una mediana de 4,75 y un promedio total de 4,57 $\pm 0,50 \mathrm{y}$, el Mejoramiento de procesos constructivos (Brecha $\mathrm{N}^{\circ} 10$ ) con una mediana de 4,50 y un promedio total de $4,43 \pm 0,50$.

Seguido de la Arquitectura como respuesta a las necesidades sociales-locales (Brecha $\mathrm{N}^{\circ} 4$ ), Desarrollo arquitectónico y urbano en proceso naturales (Brecha $\mathrm{N}^{\circ}$ 8) y la Intervención sobre el patrimonio arquitectónico (Brecha $\mathrm{N}^{\circ} 5$ ), el resto de brechas con una mediana $<4,00$ se presentan en la Tabla 3.

Tabla 3. Puntuaciones finales por brecha de investigación.

\begin{tabular}{|c|c|c|c|c|c|c|}
\hline \multirow[b]{2}{*}{ Brecha $\mathrm{N}^{\mathrm{o}}$} & \multicolumn{5}{|l|}{ Total } & \multirow[b]{2}{*}{ Prioridad $\mathrm{N}^{\circ}$} \\
\hline & Media & Mín. & Máx. & $\begin{array}{l}\text { Desv. } \\
\text { Est. }\end{array}$ & Mediana & \\
\hline $1^{o}$ & 3,25 & 2 & 4 & 0,70 & 3,25 & $8^{\circ}$ \\
\hline $2^{o}$ & 3,32 & 2 & 5 & 0,85 & 3,25 & $9^{\circ}$ \\
\hline $3^{\circ}$ & 2,45 & 1 & 4 & 0,96 & 2,50 & $10^{\circ}$ \\
\hline $4^{\circ}$ & 3,92 & 2 & 5 & 0,91 & 4,25 & $3^{\circ}$ \\
\hline $5^{\circ}$ & 4,22 & 3 & 5 & 0,69 & 4,00 & $5^{\circ}$ \\
\hline $6^{\circ}$ & 4,57 & 3 & 5 & 0,50 & 4,75 & $1^{o}$ \\
\hline $7^{\circ}$ & 3,98 & 3 & 5 & 0,50 & 3,75 & $6^{\circ}$ \\
\hline $8^{\circ}$ & 4,20 & 3 & 5 & 0,61 & 4,00 & $4^{\circ}$ \\
\hline $9^{\circ}$ & 3,18 & 1 & 5 & 1,05 & 3,50 & $7^{\circ}$ \\
\hline $10^{\circ}$ & 4,43 & 3 & 5 & 0,50 & 4,50 & $2^{\circ}$ \\
\hline
\end{tabular}

\section{Conclusiones}

A continuación, se presenta la reflexión narrativa de las prioridades de investigación.

\section{Papel de la arquitectura en las catástrofes naturales}

La naturaleza se recicla periódicamente como proceso de depuración propio del ciclo de vida de la flora y la fauna. Estos eventos cobran mayor significación cuando se encuentran con espacios intervenidos por el ser humano. Los efectos causados por la presencia de eventos naturales en zonas que han sufrido procesos antrópicos generan, en función de su intensidad, diferentes grados de damnificados tanto en zonas rurales como urbanas 11.

En las últimas dos décadas el mundo ha pasado de pertenecer de lógicas productivas y económicas rurales, basadas en actividades agropecuarias a sistemas económicos industriales y de servicios. Más del $60 \%$ de la población mundial vive hoy en día en zonas urbanas, espacios densos que dan una cualidad de alta vulnerabilidad por motivos naturales o artificiales. 
La arquitectura en este contexto juega un rol determinante para la mitigación de efectos negativos ante eventos de catástrofes naturales. Su incapacidad de responder a situaciones extremas trae consigo efectos económicos y sociales que representan pesados lastres para las sociedades y sus espacios de desarrollo (en el caso de economías emergentes), así como, frenos a las dinámicas económicas del llamado primer mundo12.

Se hace necesario generar espacios de inversión y gasto económico, en los campos de la generación de conocimiento de tipologías habitativas edificables, que recorran y exploren los senderos de la investigación en tecnologías constructivas13. Este campo disciplinario debe hacer énfasis tanto en las low-tech como en las high-tech, dependiendo los casos, contextos y territorios existentes.

\section{Mejoramiento de procesos constructivos}

La arquitectura ha sufrido a lo largo de sus diferentes momentos históricos puntos de inflexión que han determinado los senderos por los que recorrerían las diferentes respuestas a los problemas que debían ser enfrentados en distintos periodos de la humanidad. Es importante señalar y detenerse brevemente en el Renacimiento.

Periodo éste, caracterizado por devolver al ser humano su sitial en la base del pensamiento, conocimiento y ciencia, en los espacios filosóficos, sociales, culturales, entre otros.

La aparición de la perspectiva significó para la arquitectura un cambio fundamental entre el arquitecto "pensador" y el "constructor"14. El primero tiene como función la generación mental y teórico de lo que la arquitectura debería ser. Aparece la arquitectura de las ideas, la de papel, la que se planifica y se diseña desde aproximaciones teóricas cargadas de un fuerte peso histórico (como referente de la arquitectura).

El segundo modifica radicalmente la función del constructor. No, es más, necesariamente, el creador de la obra arquitectónica, sino más bien se constituye en el profesional que materializa y edifica las ideas del arquitecto. Aparecen disciplinas como la ingeniería edil, perfil de un profesional que tiene como función dominar las técnicas constructivas con las cuales se construyen los edificios.

Estas características de los dos perfiles señalados en los párrafos anteriores tienen actualmente puntos de convergencia en el mundo digital y análogo. Vale señalar que los procesos constructivos en la actualidad, que las aplicaciones de nuevas técnicas constructivas, están determinadas por el avance tecnológico del campo de la construcción.

Los avances científicos en este sentido tienen inicio en los sistemas de representación gráficos generados para resolver problemas de geometrías complejas (arquitectura paramétrica) así como software desarrollados para resolver problemas inherentes a los procesos constructivos desde el computador BIM (Building Información Modeling)15. Esta nueva manera de entender la producción edilicia ha comportado modificaciones en los "procesos en obra". 
La revolución industrial produjo al sector de la arquitectura y la construcción cambios en las técnicas constructivas. Introdujo la estandarización constructiva, es decir, pasó de una arquitectura como oficio o legado a la tradición constructiva hacia una arquitectura en serie y prefabricada. Hoy día la construcción se dota de materiales y sistemas constructivos que parten de principios de homogenización. Se trata por lo tanto de entender la versatilidad de la arquitectura partiendo de sistemas constructivos que garanticen no solamente un ahorro en los tiempos de la edificabilidad, sino que también generen ahorros en los costos económicos para las diferentes etapas del proceso constructivo hasta la obtención del producto final.

\section{Intervención sobre el patrimonio arquitectónico}

Los procesos de consolidación física de las ciudades alrededor del mundo han sido ciclos dentro de la historia de la arquitectura que han atravesado diferentes periodos. Cada uno de ellos ha respondido a modelos espaciales que fueron determinados por aspectos sociales, culturales, económicos, políticos, entre otros.

Para la cultura occidental, el paso del tiempo sobre un objeto hace que cobre un valor específico en su relación dialéctica significante-significado. La arquitectura como producción edificada de las diferentes culturas y sociedades a través del tiempo es la evidencia tangible privilegiada de la transformación de los territorios a lo largo de la Historia de la Humanidad.

Desde el año 1978, momento en el cual la UNESCO declara a las ciudades de Quito y Varsovia (Ecuador y Polonia, respectivamente) como ciudades patrimonio de la Humanidad16, se inaugura una etapa cargada de aproximaciones teóricas sobre la conservación de objetos arquitectónicos, como señal de la herencia cultural las diferentes civilizaciones que las representan.

Las distintas ciudades alrededor del mundo se han juntado a través de instituciones para la generación de políticas y gestión de espacios patrimoniales. Es el caso de la Organización de las Ciudades Patrimonio Mundial - OCPM17, organización no gubernamental sin fines de lucro que tiene como finalidad la concientización del valor que comporta la protección de ciudades patrimoniales.

Espacios como éste se encuentran funcionando de la mano de los gobiernos locales, no solamente desde una visión conservacionista del hecho edificado, sino como estrategia de desarrollo económico de las ciudades (marketing urbano) para encaminar a modelos sostenibles de desarrollo de bajo impacto ambiental.

Las ciudades se ven como oportunidades para construir sistemas económicos a diferentes escalas, desde dinámicas sociales y culturales locales, que se inserten en las ofertas mundiales de transferencia de conocimiento, desmitificando la amenaza de la homogenización de la cultura en un contexto global. Se apuesta a la heterogeneidad de los territorios al alcance de una población mundial que tiene cada vez más fácil el acceso a nuevas culturas desde los "mass media" y las redes de comunicaciones físicas y virtuales.

\section{Desarrollo urbano arquitectónico en procesos naturales}


Los territorios que han sido colonizados por el ser humano se han visto afectados en varios aspectos de los ciclos naturales con los que los ecosistemas han funcionado desde siempre.

La huella ecológica que la presencia humana ha generado en la naturaleza rompe con el justo equilibrio que existió de siempre entre el mundo animal y el resto de mundos que componen la vida en el planeta18.

Los asentamientos humanos, los poblados y ciudades constituyen sin lugar a dudas la mejor expresión del intento del ser humano por imponer su presencia en la naturaleza y, hasta en cierto momento de la historia de la humanidad, la intención de racionalizarla.

El planeta vive en estas últimas décadas un acelerado proceso de cambios climáticos, los mismos que se manifiestan en diferentes partes de los cinco continentes con comportamientos atípicos para ciertas zonas geográficas.

En esa dualidad que existe entre arquitectura y ciudad, en la que la primera consolida el espacio físico de la segunda y ésta a su vez determina la forma de la primera, hay una obligación de ambas en generar respuestas materializadas, de acciones concretas, para reducir las afectaciones sobre los territorios naturales que las sostienen.

No se trata solamente de que sean capaces de reducir el consumo energético propio de sus estructuras y de generar energía a través de sistemas pasivos, sino que es importante que constituyan en elementos configurantes del engranaje sinérgico, característico de los ciclos propios de la naturaleza19.

Para tal enfoque es necesario entender que las diferentes disciplinas que configuran ciudad, arquitectura y edificaciones tomen en consideración aspectos tecnológicos capaces de convertirse en verdaderas fuentes de desahogo para el medio ambiente y que, además, sean viables desde la inversión de recursos económicos, los mismos que son finitos, más allá de las diferentes economías mundiales de las cuales provengan.

\section{Espacio público y arquitectura}

Cuando se trata de morfología urbana, se debe hacer mención a tres factores que la configuran; i) el territorio: entendido como el espacio físico natural sobre el cual se asentará un determinado poblado o ciudad. Entran en juego los aspectos topográficos, altimétricos, orográficos, entre otros, ii) el trazado: siendo este estrato el primer componente artificial que define la forma de la ciudad.

Desde Mileto en la antigua Grecia, pasando por las ciudades medievales de Europa Occidental y atravesando por las ciudades islámicas (Magreb y Medio Oriente), las diferentes culturas han visto definidas sus ciudades por una serie de ejes imaginarios que se transformarán, con el pasar del tiempo, en las sendas que darán la forma final de sus recorridos, iii) edificaciones: serán todas las construcciones que se colocarán y que ocuparán los polígonos resultantes de los trazados. 
La ocupación de estos espacios está a cargo de la arquitectura. Estimando un rango en la relación entre áreas edificadas y no edificadas en las ciudades, se podría llegar a establecer que un $70 \%$ del territorio lo llena la arquitectura.

En esa relación numérica, que podrá variar según el modelo espacial de ciudad, existen espacios vacíos, espacios necesarios para que se den las relaciones sociales, culturales, económicas, entre otras, que darán carácter e identidad a la sociedad que en ella se desarrolla.

Estos lugares son denominados en forma genérica como espacio público. Son de diferentes cualidades, de diversos usos, de intensidades de ocupación variantes. En el mundo clásico el Ágora era el símbolo supremo de la forma de organización política y social griega20; la Edad Media tuvo reservada en la plaza del mercado el símbolo de una sociedad mercantil de artesanos y obreros. Ya para el Renacimiento el espacio público irá preparando el camino para las grandes reivindicaciones sociales que tuvo su culminen en la revolución francesa.

En la actualidad el espacio público juega un rol indispensable en la vida de los pueblos: la primavera árabe, los indignados en España, los forajidos en Ecuador; todos estos eventos urbanos socio-políticos que tuvieron como escenario diferentes ciudades y sus espacios públicos. Como telones de fondo las arquitecturas y sus intersticios que permiten la vida entre edificios 21 .

Construir sociedades con identidad en un mundo mediatizado, donde lo local juega permanentemente con lo global, en la cual la arquitectura y los espacios públicos que ésta genera entre sus paredes, cambian a velocidades vertiginosas, tratar de encontrar respuestas a los problemas contemporáneos es un desafío para los arquitectos comprometidos e implicados con la sociedad, la cultura y la educación.

\section{Agradecimientos}

La sesión fue realizada dentro del I Taller sobre Investigación Científica en Arquitectura organizado por la Dirección de Investigación e Innovación de la Universidad Internacional SEK. Agradecemos expresamente al grupo de docentes de la Facultad Arquitectura e Ingenierías por su participación.

\section{Bibliografía}

Agosin M. Crecimiento y diversificación de las exportaciones en economías emergentes. Revista. ES CONFERENCIA. Disponible en: www.cieplan.org/media/publicaciones/archivos/302/Paper_de_Manuel_Agosin_Decano_ Fac_de_Economia_U_de_Chile.pdf

Doménech Quesada JL. (2007). Huella Ecológica y Desarrollo sostenible, AENOR.

Ecuador. Reglamento General de la Ley Orgánica de Educación Superior. Registro Oficial No. 526. República del Ecuador; 2011. 
Ecuador. Reglamento de Régimen Académico. Registro Oficial No. 136. República del Ecuador; 2013.

Ecuador. Plan Nacional para el Buen Vivir 2013-2017. Secretaría Nacional de Planificación y Desarrollo. República del Ecuador; 2013.

Fink A, Kosecoff J, Chassin M, Brook RH. Consensus methods: characteristics and guidelines for use. Am J Public Health. 1984; 74: 979-983.

Gómez García AR, Suasnavas Bermúdez PR, Vilaret Serpa A, Silva Peñaherrera MG, Russo Puga M. Propuesta metodológica para el establecimiento de líneas de investigación en seguridad y salud en el trabajo. Innova Research Journal. 2016; 1(9): 13-25.

Jan Gehl. (2006). La humanización del espacio urbano: la vida entre edificios, Reverté.

Landeta J. 1999. El método Delphi. Barcelona, España. Ariel.

Martín Hernández M. (2014). La casa en la arquitectura moderna. Reverté.

Montaner JM. (2015). La Condición contemporánea de la arquitectura. GG.

Morláns MC, Introducción a la Ecología del Paisaje, Editorial Científica UniversitariaUniversidad Nacional de Catamarca, S.F. del V. de Catamarca, Argentina, 2005.

Neila J. (2000). Arquitectura Bioclimática en un entorno sostenible: buenas prácticas edificatorias. Boletín $\mathrm{CF}+\mathrm{S} n^{\circ} 14$, Madrid.

Organization of World Heritage Cities. Historic cities, memory of the world. Disponible en: http://www.ovpm.org

Quero Virla M. Confiabilidad y coeficiente Alpha de Cronbach. Telos. 2010; 12: 248-252.

Romero L, Quental C. Métodos de priorización de investigación para la salud: su revisión como instrumento para la construcción de la agenda nacional en Panamá. Invest. Pens. Crit. 2014; $2(5) ; 34-50$.

Sánchez LM. (2016). Centro Histórico de Quito: aportes para reflexionar sobre la preservación de las casas patio desde el estado de concientización usuaria.

Sandra M. Revistas y producción científica de América Latina y el Caribe: su visibilidad en SciELO, RedALyC y SCOPUS. Revista Interamericana de Bibliotecología. 2011; 34: 187 199.

Van de Ven AH, Delbecq AL. The Nominal group as a research instrument for exploratory health studies. Am J Publ Health. 1972; 62: 337-342. 
Vera Aranda AL. (2015). Breve Historia de las ciudades del Mundo Antiguo. Edicionaes Nowtilus.

Wiebenson D, Blume H. (1988). Los tratados de arquitectura: de Alberti a Ledoux. 